\title{
Grasping in the Pigeon: Control Through Sound and Vibration Feedback Mediated by the Nucleus Basalis
}

\author{
ULRICH SCHALL AND JUAN D. DELIUS
}

\author{
Allgemeine Psychologie, Universität Konstanz, D-7750 Konstanz, Germany
}

\begin{abstract}
Pigeons were trained to detect auditory and vibratory stimuli in two separate experiments using an instrumental conditioning procedure. The discriminative stimuli became effective as the subjects grasped a probe with the beak. The pigeons learned to suppress responding upon this grasp-contingent stimulation. Bilateral lesions of the nucleus basalis prosencephali (Bas), known to be involved in the motor control of pecking and to receive short latency input of cochlear and trigeminal origin, eliminated the behavioral stimulus detection. The performance of a control color discrimination was not affected by the Bas lesions, demonstrating that these had a specific effect. The processing of peck-related feedback by the nucleus basalis during the normal food uptake of pigeons is discussed.
\end{abstract}

Pigeon Pecking Grasping $\begin{gathered}\text { Sound } \\ \text { Nucleus basalis prosencephali }\end{gathered}$ Lesions

THE control mechanisms of grasping motions by organisms have recently received increased attention $(12,18)$. This interest is partly motivated by their potential role as models for robot manipulators. The precise role of sensory input for the patterning of such motor responses is still a controversial issue. This applies particularly to the effects of mechanical stimuli generated by the movements themselves (reafferences). Thus far research has focused on the primate/human arm and hand and other animal grasping systems have been relatively neglected. The avian neck, head and beak is a system that could provide relevant insights (16). The pecking, grasping, mandibulating and swallowing of several bird species have been described in some detail $(33,34)$ and information about the neural substrates controlling these movements is beginning to accumulate $(2,3,10,19)$.

In the course of normal feeding, the pecking of pigeons has a series of mechanical consequences, all of which could in principle provide useful reafferent information. To begin with, there are proprioceptive signals from muscles and joints $(15,23)$. There is inevitably also vestibular stimulation elicited by the motion of the head (26). Then there are mechanical events arising from the contact of the beak with the substrate during impact and prehension. These give rise to several potential stimuli. For example, airborne sound and bone-conducted sound that could excite cochlear hair cells. Bone transmission is likely to be aided by the fact that in birds the tympanum is in part suspended from the quadratum, a component of the bony jaw apparatus (27). Tissue-conducted vibration, however, must also excite mechanoreceptors. The bird's beak is richly furnished with Herbst corpuscles known to be particularly sensitive to vibration $(9,11,21)$. Subsequent mandibulation and swallowing of food morsels very probably stimulates these and other mechanoreceptors.
Several studies suggest that the Bas, a structure peculiar to the anterior forebrain of sauropsids (reptiles and birds), is involved in the control of avian feeding, more precisely in the control of pecking $(6,31)$. Anatomical evidence indicates that the Bas receives, among other inputs, paucisynaptic afferents of trigeminal and cochlear origin $(1,29)$. Electrophysiological findings confirm that the Bas responds with short latency to tactile stimulation of the beak and to acoustic stimulation of the cochlea $(20,27,28,32)$. Therefore, it has been suggested that both auditory and vibratory stimuli arising from the contact of the beak with the substrate may provide feedback signals that influence the course of pecking activity $(3,24)$. The findings we now describe demonstrate that the Bas does indeed participate in the modulation of this behavior depending on vibratory and auditory feedback information. In two separate experiments, pigeons were first trained to grasp a target for food reward and then differentially conditioned to refrain from grasping when this response yielded a vibratory or an auditory feedback signal. The subjects' performance was reexamined after bilateral Bas ablations. A conditioned color discrimination served as a concurrent comparison task.

METHOD

\section{Vibration Detection}

Subjects. Homing pigeons (Columba livia) of local stock were used. They were housed in a brightly lit $(12 \mathrm{~h}$ on, $12 \mathrm{~h}$ off) and well-ventilated animal room. For the duration of the experiment they were kept food deprived to $80 \%$ of their ad lib feeding weight. 


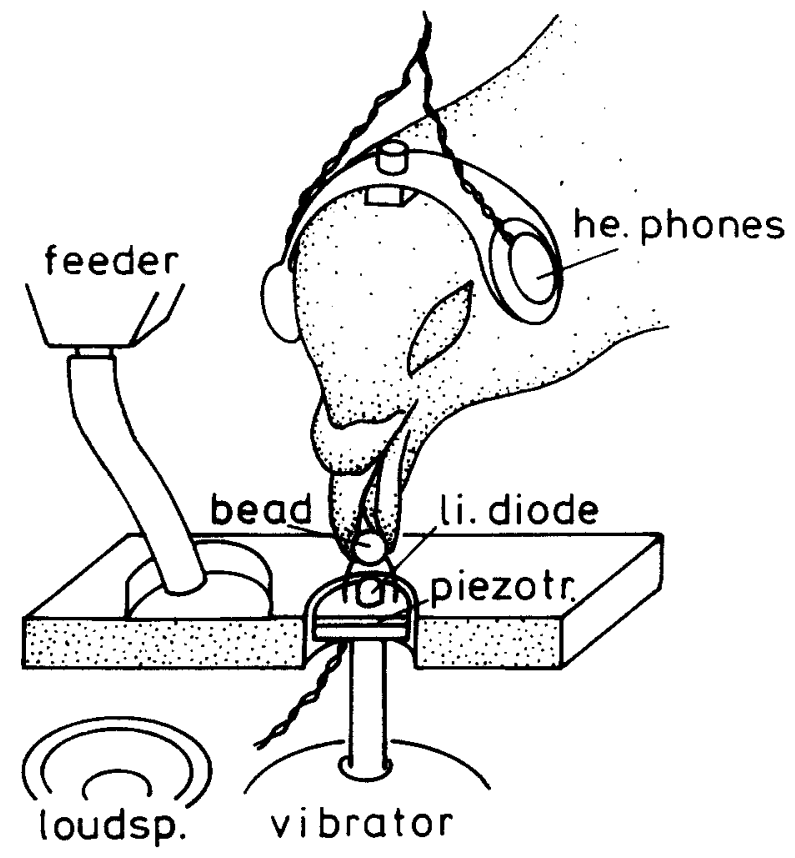

FIG. 1. Schematic diagram of the apparatus (see text for explanations).

Apparatus. A modified cage $(40 \times 50 \times 45 \mathrm{~cm})$ placed in a separate experimental cubicle served as an experimental chamber. A metal ledge $(15 \times 6 \mathrm{~cm})$ located $5 \mathrm{~cm}$ above the cage floor replaced the normal feeding trough (Fig. 1). A vibrator unit (Ling) was positioned below it. The stylus of the vibrator, bearing a circular piezoelectric transducer (Valvo, $12 \mathrm{~mm}$ dia.), protruded through an opening in the ledge. A two-color lightemitting diode ( $3 \mathrm{~mm}$ dia.) and a wooden bead ( $6 \mathrm{~mm}$ dia.), the latter mounted on a small wire bridge above the diode, were glued onto the transducer. Next to this stimulating/sensing probe was a circular trough $(2.5 \mathrm{~cm}$ dia., $0.6 \mathrm{~cm}$ deep). The delivery tube of an electromagnetic feeder terminated in the trough. When activated by a pulse, the feeder issued a few grains of millet.

The instrumental response demanded from the pigeons was a pecking and grasping of the bead. The piezotransducer signals generated by this activity were amplified and fed into a threshold circuit. The stimuli to be detected were either vertical sinusoidal motions of the probe $(125 \mathrm{~Hz}, 50 \mu \mathrm{m}$ peak to peak amplitude) or red light emitted by the diode. The vibrator was driven by a gated oscillator (trapezoidal envelope, $5 \mathrm{~ms}$ rise and fall). A proximity transducer (Vibrometrics) built into the vibrator unit and connected to an oscilloscope (Tektronix) and an electronic counter (Racal) were used to monitor the amplitude and frequency of the vibration. Since the vibrator also produced a low intensity but just audible hum (55 dB SPL measured with a Rhode and Schwartz phonometer $5 \mathrm{~cm}$ above the ledge) when activated, control sessions were run with a pure sound stimulus of identical frequency and equivalent loudness. The hum was emitted by a loudspeaker $(4 \mathrm{~W}, 10 \mathrm{~cm}$ dia.) placed next to the vibrator and driven by the suitably readjusted oscillator. All experimental events were controlled and recorded by programmable modular digital/analogue equipment (Massey-Dickinson).

Procedure. In daily sessions lasting up to $45 \mathrm{~min}$, the subjects were first shaped to peck and then to grasp the bead for grain reinforcement. The green diode light was on continuously. Pecks at the bead occurred spontaneously after a few free deliv- eries of grain. Such pecks automatically triggered reward. When pecking was established, the sensitivity of the transducer system was decreased, forcing the animals to grasp and tug the bead for reinforcement. Following this, the animals were gradually trained to grasp repeatedly before receiving a reward. When they mastered a fixed ratio schedule of 10 responses per reinforcement and managed to obtain about 200 reinforcements within $15 \mathrm{~min}$ they were transferred to the vibration detection procedure.

The daily vibration detection sessions consisted of 48 positive and 48 negative quasirandomly sequenced trials (8). All trials began with the onset of the green diode light. During positive trials the vibrator remained inactive. The second of two grasping responses initiated a scoring period lasting $3 \mathrm{~s}$ during which all further grasps were counted. When 3 or more additional responses occurred during this period, the animal was rewarded before the end of the scoring period and trial. During negative trials, the vibrator was switched on by the first grasp and remained on until the end of the trial. If the animal refrained from further responding after a second grasp the trial terminated after $5 \mathrm{~s}$. Further grasps reinitiated the 5-s delay. Thus negative trials ended only when the animal had not responded for $5 \mathrm{~s}$. However, as in positive trials, responses were only scored during the $3 \mathrm{~s}$ following the two first grasps. Note that the probe vibration could only be sensed by the subjects while they contacted the target bead with the bill. All trials terminated with a $7-\mathrm{s}$ intertrial interval during which the piezotransducer was disabled, the light diode was off, and no vibratory stimulus was present. Control sessions served to check that the hum produced by the vibrator did not act as an artifactual cue. In these, the loudspeaker replaced the vibrator as cue source. Otherwise the procedure was exactly as described above.

The detection of red light served as a comparison task. The corresponding sessions were structured just as those described above, except that the first grasp during negative trials caused the diode's color to change from green to red. It remained in that state until the end of the trial. Both vibrator and loudspeaker were inactive.

When more than one kind of session was run on the same day, they were separated by at least one hour. The order was randomized over days.

Surgery. When the animals had completed training, they received bilateral Bas lesions. They were anesthetized with a pentobarbital/chloral-hydrate mixture administered intramuscularly (22). While their head was held in a stereotactic apparatus, the scalp was incised and the cranial bone was trephined above the target areas. A stainless steel electrode $(0.15 \mathrm{~mm}$ dia., $0.5 \mathrm{~mm}$ uninsulated sharpened tip) was lowered so that the tip was within the Bas according to the standard pigeon brain atlas (13) (coordinates A $12, \mathrm{~L} 3.5, \mathrm{~V} 7$ ). A $30 \mathrm{~mA}$ high frequency current (Radionics coagulator) was applied for $30 \mathrm{~s}$. Three further coagulations were performed $0.5 \mathrm{~mm}$ away from the initial placement in an attempt to destroy all of the Bas. Both Bas were lesioned according to the same scheme. After the final withdrawal of the electrode, the wound was closed and the animals treated with an antibiotic. A recovery period of 4 days was allowed before the animals were tested again.

Histology. When the behavioral experiments were complete, the animals were anesthetized and perfused transcardially with saline $(0.9 \%)$ and formalin (4\%) solutions. After a few days postfixation, the brains were extracted and embedded in egg yolk hardened with formalin vapors. Sections $(40 \mu \mathrm{m})$ covering the relevant portion of frontal forebrain were stained with cresyl-violet. The location and extent of the lesions were determined with the aid of a microscope and transferred onto diagrams derived from the brain atlas (13). 


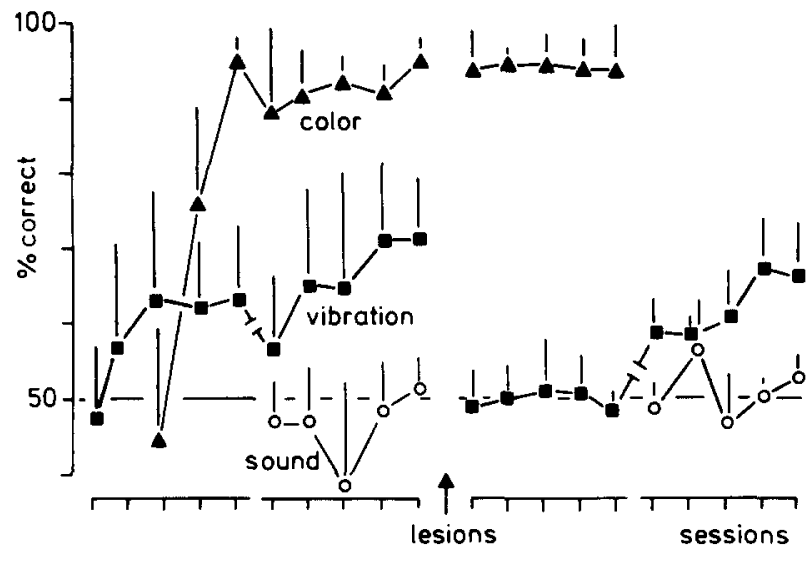

FIG. 2. Mean pre- and postoperative vibration detection performance of 3 pigeons. Also shown is the mean performance on a comparison color detection task and a control sound detection task. For brevity the middle 5 pre- and postoperative vibration sessions have been left out. Arrow indicates bilateral Bas lesions.

\section{RESULTS}

Response shaping was completed within 5 sessions. Preoperative vibration detection training sessions ran for 15 days. The last 5 days also included sound control sessions. The color detection training ran concurrently during the last 8 preoperative days. Performance on each task was expressed as the percent correct responses (those counted during the scoring periods corresponding to positive trials) over the total number of responses (those counted during all scoring periods) of a session.

Though all 6 experimental birds gave evidence of leaming the vibration detection task, only 3 achieved the criterion of two final sessions with $70 \%$ or more correct responses. The data that we now report concerns only these birds. They showed a steady improvement over the training sessions (Fig. 2). During the last 5 sessions their performance was significantly above chance [analysis of variance for repeated measures (14) on the absolute number of correct and incorrect responses per session, $F(1,18)=$ $46.81, p<0.01$ ]. The 5 control sound detection sessions yielded chance results, $\mathrm{F}(1,18)=1.2, p>0.05$. The color discrimination was learned very rapidly and performance was well above chance over the final 5 sessions, $\mathrm{F}(1,18)=209.3, p<0.001$.

Following the Bas lesions the 3 pigeons were retrained for 15 days on the vibration detection task. The color discrimination was retrained during only the first 5 days. The last 5 days included further sound control sessions. Vibration detection performance was markedly impaired by the lesions [comparison 5 last preoperative/ 5 first postoperative sessions, $F(1,18)=173.0$, $p<0.001]$. It actually fell back to chance level after the surgery, $F(1,18)=3.5, p>0.05$, but recovered somewhat towards the end of retraining [ 5 final sessions, $\mathrm{F}(1,18)=18.8, p<0.01$ ]. The color discrimination, by contrast, was not affected [ 5 last preop $/ 5$ first postop sessions, $\mathrm{F}(1,18)=2.1, p>0.05$ ]. Control sound detection never exceeded chance levels, $F(1,18)<1.18, p>0.05$, proving that the vibration detection was not mediated by the vibrator's hum artifact.

Histology showed that in all 3 birds the coagulations had destroyed the posterior and main part of both right and left Bas (Fig. 3). The terminal portions of the quinto-frontal tracts (the main afferent pathways of the Bas) were largely transected. The surrounding neostriatum frontale (which is anatomically closely related to the Bas) and the stem of the fronto-achistriatal tracts [the main efferent pathways of the Bas system (29)] were par- tially destroyed. The overlying most anterior tips of the ectostriatum were also slightly affected.

A discussion of these results is postponed until the next experiment dealing with acoustic stimulation has been described.

\section{METHOD}

\section{Tone Detection}

New pigeons of the same origin were used. They were housed and food deprived as already described. Prior to training, each had a small tapped metal block $(3.5 \times 6 \times 1.5 \mathrm{~mm})$ attached to their cranium with dental cement, following the general surgical procedures described previously. During training sessions the birds wore a pair of miniature earphones (Sony) glued to a small metal bow. The latter was fixed with a screw to the pigeons' head blocks. The headphones were connected via a hearing aid cable suspended from an elastic thread to the gated oscillator. The apparatus was otherwise the same as in the previous experiment except that the vibrator and loudspeaker were not used. The subjects were shaped as in the preceding experiment. The tone detection task used the same procedure as the vibration detection task previously described. However, the stimuli to be detected were sinusoidal tone pulses of $5 \mathrm{kHz}$ and 65 $\mathrm{dB}$ (SPL, measured between the earphones) lasting $250 \mathrm{~ms}$. Each bead grasp during the negative trials triggered one such pulse. Note that the quality (higher intensity, higher frequency) and the delivery (earphone presentation, response contingent) of the present acoustic stimulus differed markedly from that employed in the sound artifact control task of the previous experiment. The same red light detection task as in the previous experiment served as a comparison task. Bilateral Bas coagulations were placed following the same procedure described earlier. The histological techniques were also identical.

\section{RESULTS}

Pretraining was again complete after 5 sessions. Even though all 6 pigeons showed some evidence of learning the tone detection task, only 3 achieved the criterion of 2 final sessions with $70 \%$ or more responses correct within the 10 prescheduled training sessions (Fig. 4). All results reported refer to these successful subjects. Their performance during the last 5 tone detection sessions was significantly above chance level, $F(1,18)=39.88$, $p<0.01$. The color detection task was run on the same days as the latter. It was rapidly learned and the overall performance over the 5 sessions was well above chance, $F(1,18)=83.8$, $p<0.01$.

The Bas lesions led to a marked deterioration of the tone detection performance $[5$ last preop/ 5 first postop sessions, $F(1,18)=$ $35.6, p<0.01]$. The color discrimination that was run concurrently on the same days was not affected $[5$ preop/5 postop sessions, $\mathrm{F}(1,18)=1.11, p>0.05]$. There was hardly any recovery in performance during the 5 further daily sessions of tone detection. Performance on this task remained at chance level throughout all 10 postoperative sessions, $\mathrm{F}(1,18)<2.8, p>0.05$.

Histological examination revealed lesions of the Bas area that were closely similar to those of the previous experiment, except that in one bird the lesions were placed slightly more anteriorly, destroying most of the Bas, but completely sparing the ectostriatum (Fig. 3). Its behavioral output, however, had not differed from that of the other birds.

\section{DISCUSSION}

The two experiments reported were firstly concerned with the role that acoustic and vibratory stimuli arising during the grasp- 

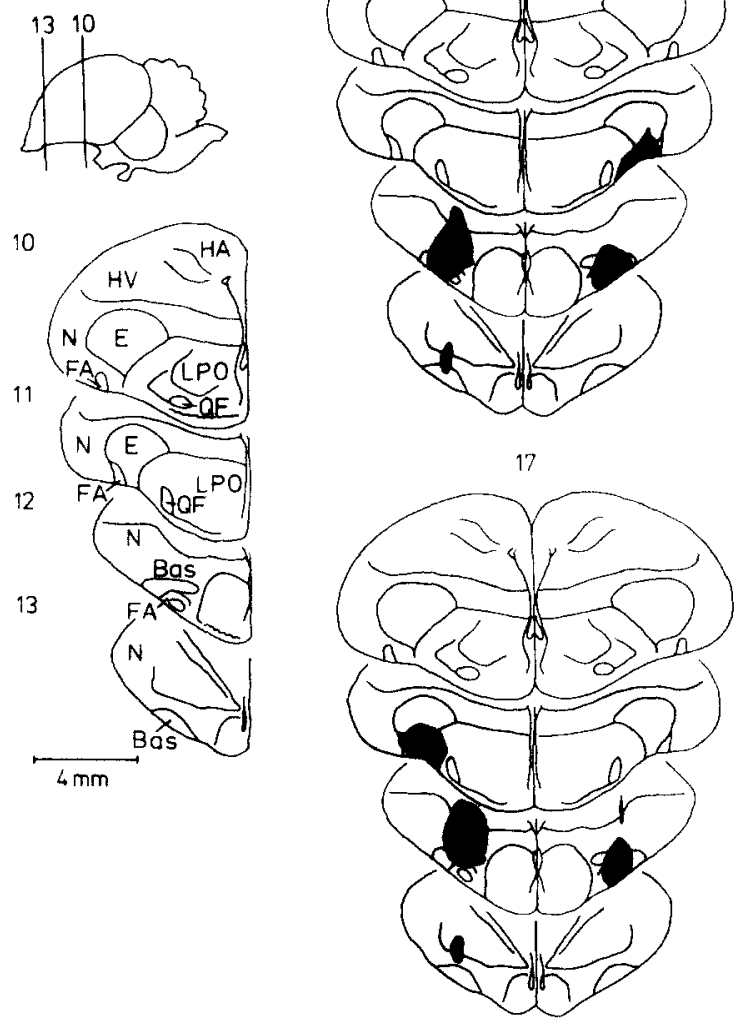

$\mathrm{T}$
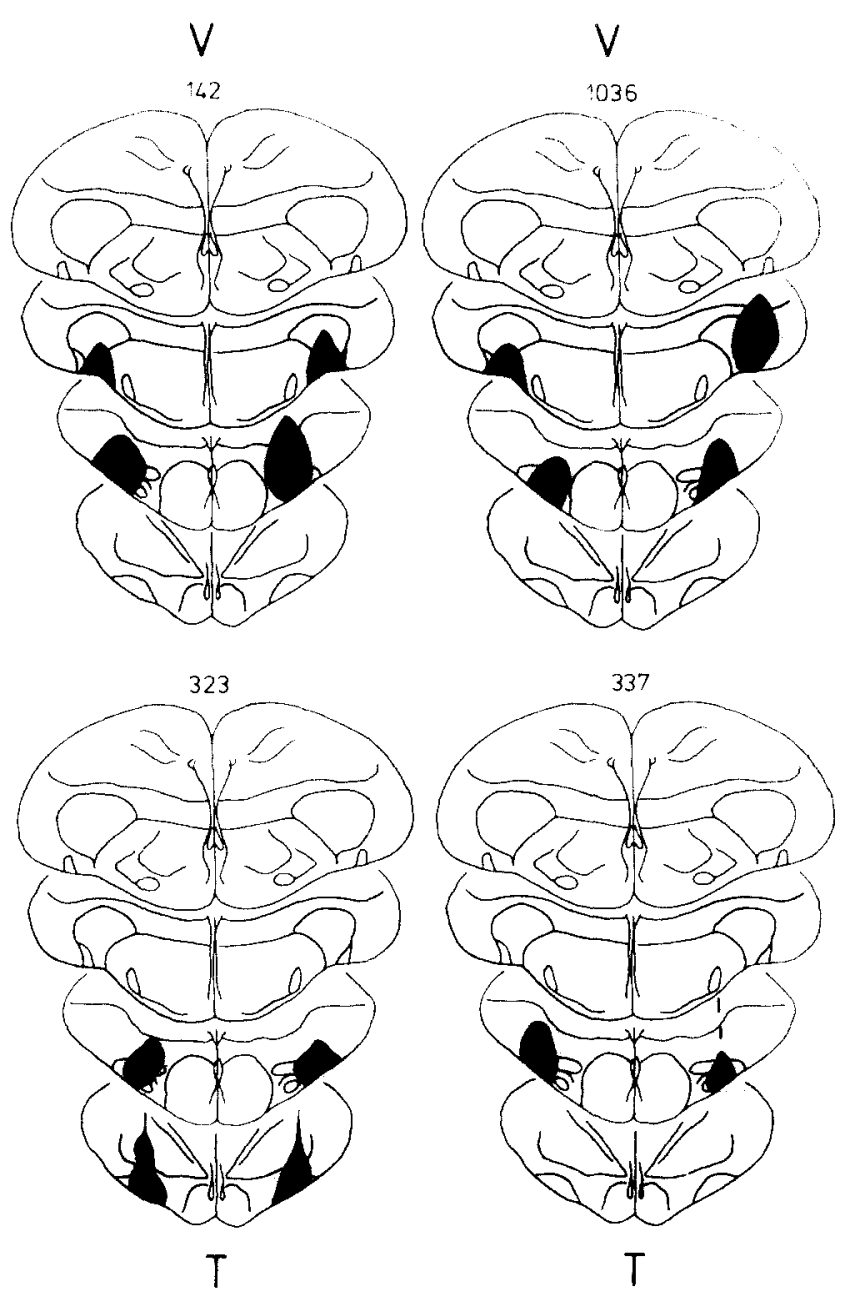

FIG. 3. Lesions of the nucleus basalis (Bas) and surrounding structures in 3 pigeons taking part in the vibration detection experiment $(\mathrm{V}$, top) and in 3 pigeons participating in the tone discrimination experiment ( $\mathrm{T}$, bottom). Other abbreviations: $\mathrm{E}$, ectostriatum; $\mathrm{FA}$, tractus fronto-archistriaticus; $\mathrm{HA}$, hyperstriatum accessorium; HV, h. ventrale; LPO, lobus paraolfactorius; $\mathrm{N}$, neostriatum; QF, t. quinto-frontalis.

ing phase of pecking might have in determining the further course of foraging behavior in pigeons. Grasp-probe vibration and headphone tones delivered contingent upon beak prehension were shown to be capable of exerting reliable conditioned inhibition over a food-rewarded peck/grasp response. Stimuli of equivalent qualities that are known to arise during normal feeding are thus likely to be significant feedback signals during normal alimentary pecking. The less than faultless detection of the experimental tone and vibration stimuli achieved within the admittedly not very extensive training is in turn likely to have been due to the perceptual masking caused by these unavoidable, natural auditory/somaesthetic consequences of beak/target contacts.

The much better performance on the visual comparison task is likely to have been due both to the absence of comparably competing stimulation and to the more extended response independent presentation of the relevant discriminative cue. Some evidence, however, indicates that auditory stimuli, differently from visual ones, are in fact most effective in gaining control over food-rewarded pecking in pigeons when they are presented in feedback-like contingency with pecks $(3,4,7)$. This is congruent with the fact that in a granivorous bird, only peck-elicited sound (and also vibration) is likely to be informative about food availability or quality. In contrast, brief visual stimuli immediately following pecking turn out to be ineffective discriminatory cues. This is almost certainly due to the fact that pigeons reflexively close their eyes just before and during beak-substrate impact (3).

While it is reasonably certain that the headphone-delivered tones exclusively excited cochlear receptors, there can be less certainty about which receptors were excited by the vibration acting on the beak-tip. Palate mechanoreceptors, skeleto-muscular proprioceptors and cochlear hair cells are all possible candidates. Since vibration detection was abolished by Bas lesions and the Bas is not known to receive input from proprioceptors, it is, however, unlikely that these played a role in our experiment. The involvement of the cochlea through tissue-conducted vibration cannot, however, be dismissed. The pigeon's cochlea is singularly sensitive to low frequency waves $(17,28)$. It can, however, be assumed that due to their proximity to the stimulus source and their extreme sensitivity, palate (Herbst) mechanoreceptors were massively involved $(9,27)$. This conclusion is further supported by a report that trigeminal deafferentations markedly disturb the feeding behavior of pigeons (24).

The experiments were also concerned with the likelihood that the nucleus basalis prosencephali mediates effects of feedback 


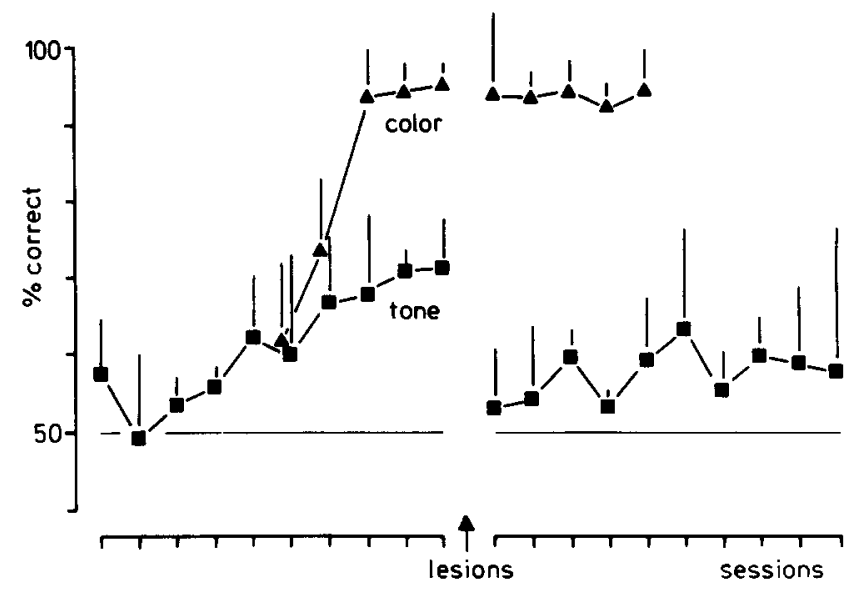

FIG. 4. Mean pre- and postoperative tone detection performance of 3 pigeons. Also illustrated, performance on a comparison color detection task. Arrow indicates a bilateral Bas coagulation.

tone and vibration stimuli upon pecking behavior. The Bas, as already mentioned, is known to receive short latency mechanoreceptive input from the palate and the cochlea via, respectively, the main sensory nucleus of the fifth nerve and the intermediate nucleus of the lateral lemniscus $(1,26-30)$. Our results show that in accordance with these circumstances, bilateral Bas lesions practically abolish the discriminative control over pecking of auditory and vibratory stimuli consequent upon grasping.

The finding that the color discrimination was not at all affected by Bas lesions in either experiment suggests that the above mentioned deficits were specific and not simply due to motor impairment or general trauma. That the relative ease of the visual task could have made it generally impervious to lesions is unlikely, as ablations of the lateral neostriatum, for example, have been found to strongly impair performance of a comparable color task (5). The fact that the present Bas lesions had no effect is rather in agreement with the lack of any substantial, visual projections to the Bas (29).

That there was still some vibration and tone detection after the lesions can be possibly ascribed to the fact that additional auditory and somatosensory projection areas exist in the pigeon's brain (notably the so-called area $\mathrm{L}$ in the mediocaudal neostriatum) (25). None of these, however, are known to be directly involved with the control of pecking, and they may thus only function as fall back systems. Regardless of that, the present findings further the assumption that the Bas is importantly involved in the finer sensory-motor coordination of the later phases of pecking $(3,33)$. This has been sometimes queried on the ground that Bas lesions often do not impair the gross execution of alimentary pecking $(19,27)$. Future work will have to determine more precisely which aspects of peck-produced stimuli contribute towards the shaping of which components of the peck action pattern, and to find out exactly how the avian nucleus basalis prosencephali mediates such coordinations.

\section{ACKNOWLEDGEMENTS}

The research was supported by the Deutsche Forschungsgemeinschaft. We are grateful to A. Lohmann, J. Frettlöh, Drs. R. Jäger and O. Güntürkün for much assistance. We also thank M. Siemann, A. Franchini, Drs. W. Hörster and C. Wynne for essential help with manuscript preparation.

\section{REFERENCES}

1. Arends, J. J. A.; Zeigler, H. P. Anatomical identification of an auditory pathway from a nucleus of the lateral lemniscal system to the frontal telencephalon (nucleus basalis) of the pigeon. Brain Res. 398:375-381; 1986.

2. Berkhoudt, H.; Klein, B. G.; Zeigler, H. P. Afferents to the trigeminal and fascial motor nuclei in pigeon (Columba livia): Central connections of jaw motorneurons. J. Comp. Neurol. 209:301$312 ; 1982$.

3. Delius, J. D. The pecking of the pigeon: Free for all. In: Lowe, C. F.; Richelle, M.; Blackman, D. E.; Bradshaw, C. M., eds. Behaviour analysis and contemporary psychology. Hillsdale, NJ: Erlbaum; 1985:53-81.

4. Delius, J. D.; Emmerton, J. Stimulus dependent asymmetry in classical and instrumental discrimination learning by pigeons. Psychol. Rec. 28:425-434; 1978.

5. Delius, J. D.; Jäger, R.; Friesel, M. Lateral telencephalic lesions affect visual discriminations in pigeons. Behav. Brain Res. 11:249$258 ; 1984$.

6. Delius, J. D.; Runge, T. E.; Oeckinghaus, H. Short latency auditory projections to the frontal telencephalon of the pigeon. Exp. Neurol. 63:594-609; 1979.

7. Gebauer, W. Appetitives instrumentelles Diskriminationslemen von Tönen bei Tauben. Diplomthesis, Psychology. Ruhr-Universität Bochum; 1980.

8. Gellermann, L. W. Chance orders of alternating stimuli in visual discrimination experiments. J. Gen. Psychol. 42:206-208; 1933.

9. Hillebrand, K. Untersuchungen zu Schnabelafferenzen der Taube. Diplomthesis, Biology. Ruhr-Universität Bochum; 1984.

10. Hörster, W.; Franchini, A.; Daniel, S. Organization of neck muscle motoneurons in the cervical spinal cord of the pigeon. Neuroreport 1:93-96; 1990.

11. Hörster, W.; Shen, J.; Schwartzkopff, J. Electrophysiological studies on the vibrational sensitivity in the pigeon. Naturwissenschaften 70:151-152; 1983 .
12. Jeannerod, $M$. The neural and behavioural organization of goal-directed movements. Oxford: Clarendon Press; 1988.

13. Karten, H. J.; Hodos, W. A stereotaxic atlas of the brain of the pigeon. Baltimore, MD: Johns Hopkins Press; 1967.

14. Kirk, R. Experimental design: Procedures for the behavioural sciences. Monterey, CA: Brooks/Cole Publishing; 1968.

15. Kishida, R.; Dubbeldam, J. L.; Goris, R. C. Primary sensory ganglion cells projecting to the principal trigeminal nucleus in the mallard, Anas platyrhynchos. J. Comp. Neurol. 240:171-179; 1985.

16. Klein, B. G.; Deich, J. D.; Zeigler, H. P. Grasping in the pigeon (Columba livia): Final common path mechanisms. Behav. Brain Res. 18:201-213; 1985.

17. Kreithen, M. L.; Quine, D. B. Infrasound detection by the homing pigeon, Columba livia. J. Comp. Physiol. 129:1-4; 1979.

18. Kuperstein, M. Neural model of adaptive hand-eye coordination for single postures. Science $239: 1308-1311 ; 1988$.

19. Lindenblatt, U.; Delius, J. D. Nucleus basalis prosencephali, a substrate of apomorphine-induced pecking in pigeons. Brain Res. 453: $1-8 ; 1988$

20. Maekawa, M. Auditory responses in the nucleus basalis of the pigeon. Hear. Res. 27:231-237; 1987.

21. Malinovsky, L.; Zemanek, R. Sensory innervation of the skin and mucosa of some parts of the head in the domestic fowl. Folia Morphol. 9:18-23; 1971 .

22. Mallin, H. D.; Delius, J. D. Inter- and intraocular transfer of color discriminations with mandibulation as an operant in the head-fixed pigeon. Behav. Anal. Lett. 3:297-309; 1983.

23. Manni, E.; Bortolami, R.; Azzena, G. B. Jaw muscle proprioception and mesencephalic trigeminal cells in birds. Exp. Neurol. 12: 320-328; 1965.

24. Miller, M. G.; Zeigler, H. P.; Miller, A. E. Trigeminal deafferentiation and feeding behavior in the pigeon (Columba livia). J. Comp. Physiol. Psychol. 92:1025-1040; 1978.

25. Necker, R. Somatosensory system; hearing. In: Abs, M., ed. Phys- 
iology and behaviour of the pigeon. London: Academic Press; 1983:169-219.

26. Schall, U. Vestibular, olfactory, and vibratory responses of nucleus basalis prosencephali neurons in pigeons. Neurosci. Res. 4:376-384; 1987.

27. Schall, U. Sensory control of pecking in the pigeon (Columba livia). Ph.D. thesis. Universität Konstanz; 1988.

28. Schall, U.; Delius, J. D. Sensory inputs to the nucleus basalis prosencephali, a feeding-pecking centre in the pigeon. J. Comp. Physiol. [A] 159:33-41; 1986.

29. Schall, U.; Güntürkuin, O.; Delius, J. D. Sensory projections to the nucleus basalis prosencephali of the pigeon. Cell Tissue Res. 245: 539-546; 1986.

30. Wild, J. M.; Arends, J. J.; Zeigler, H. P. A trigeminal sensorimo- tor circuit for pecking, grasping and feeding in the pigeon (Columba livia). Brain Res. 300:146-151; 1984.

31. Wild, J. M.; Arends, J. J.; Zeigler, H. P. Telencephalic connections of the trigeminal system in the pigeon (Columba livia): a trigeminal sensorimotor circuit. J. Comp. Neurol. 234:441-464; 1984.

32. Witkovsky, P.; Silver, R.; Zeigler, H. P. The nucleus basalis of the pigeon: A single unit analysis. J. Comp. Neurol. 147:119m-128; 1973.

33. Zeigler, H. P.; Levitt, P. W.; Levine, R. R. Eating in the pigeon (Columba livia): Movement patterns, stereotype, and stimulus control. J. Comp. Physiol. Psychol. 94:783-794; 1980.

34. Zweers, G. A. Pecking of the pigeon (Columba livia L.). Behaviour 81:173-230; 1982 . 